\title{
Acute Toxic Leukoencephalopathy: Etiologies, Imaging Findings, and Outcomes in 101 Patients
}

\author{
(D) C. Özütemiz, (D)S.K. Roshan, DN.J. Kroll, (D).C. Benson, DJ.B. Rykken, (DM.C. Oswood, (D) Z. Zhang, and (D)A.M. McKinney
}

\begin{abstract}
BACKGROUND AND PURPOSE: Prior studies regarding acute toxic leukoencephalopathy (ATL) are either small, or preliminary. Our aim was to evaluate etiologies of and differences in imaging severity and outcomes among various etiologies of ATL.
\end{abstract}

MATERIALS AND METHODS: MRIs of patients with suspected ATL over 15 years were retrospectively reviewed; inclusion criteria were: $\mathrm{MRI}<3$ weeks of presentation with both DWI and FLAIR. These were jointly graded by two neuroradiologists via a previously described score of severity. Clinical outcome was evaluated via both modified Rankin (mRS) and ATL outcome (ATLOS) scores, each being correlated with the DWI and FLAIR scores. Etiologic subgroups of $n>6$ patients were statistically compared.

RESULTS: Of 101 included patients, the 4 subgroups of $n>6$ were the following: chemotherapy ( $n=35)$, opiates $(n=19)$, acute hepatic encephalopathy $(n=14)$, and immunosuppressants $(n=11)$. Other causes $(n=22$ total) notably included carbon monoxide $(n=3)$ metronidazole $(n=2)$, and uremia $(n=1)$. The mean DWI/FLAIR severity scores were $2.6 / 2.3,3.3 / 3.3,2.1 / 2.1$ and 2.0/2.5 for chemotherapeutics, opiates, AHE and immunosuppressants, respectively, with significant differences in both imaging severity and outcome $(P=$ .003-.032) among subgroups, particularly immunosuppressant versus chemotherapy-related ATL and immunosuppressants versus opiates $(P=.004-.032)$ related ATL. DWI and FLAIR severity weakly correlated with outcome $(\rho=0.289-.349, P<.005)$ but correlated stronger in the chemotherapy $(\rho=0.460-.586, P<.010)$ and opiate $(\rho=.472-.608, P<.05)$ subgroups, which had the worst outcomes. ATL clinically resolved in $36 \%$, with severe outcomes in $23 \%$ (coma or death, $9 / 16$ deaths from fludarabine). Notable laboratory results were elevated CSF myelin basic protein levels in $8 / 9$ patients and serum blood urea nitrogen levels in 24/91.

CONCLUSIONS: Clinical outcomes of ATL vary on the basis of etiology, being worse in chemotherapeutic- and opiate-related ATL. Uremia may be a predisposing or exacerbating factor.

ABBREVIATIONS: $\mathrm{AHE}=$ acute hepatic/hyperammonemic encephalopathy; $\mathrm{ATL}=$ acute toxic leukoencephalopathy; $\mathrm{ATLOS}=$ acute toxic leukoencephalopathy outcome score; $\mathrm{CTL}=$ chronic toxic leukoencephalopathy; $\mathrm{MBP}=$ myelin basic protein; NAWM= normal appearing white matter; PRES = posterior reversible encephalopathy syndrome; PVWM = periventricular white matter

$\mathrm{T}$

he term "toxic leukoencephalopathy" refers to nonspecific neurologic symptoms (such as altered mental status) due to injury of the cerebral white matter, occurring from a variety of conditions. ${ }^{1-3}$ This can exist in either the acute form as acute toxic leukoencephalopathy (ATL) or in the chronic form as chronic toxic leukoencephalopathy (CTL). Notably, patients have more subtle and slower development of neurologic symptoms and MR imaging findings in CTL. ${ }^{3}$ On the other hand, ATL is a relatively uncommon condition in acute encephalopathic (i.e. obtunded) patients, particularly in those who are post-chemotherapy or im-

Received August 29, 2018; accepted after revision December 3.

From the Department of Radiology (C.Ö., S.K.R., J.C.B., J.B.R., A.M.M.) and Faculty of Medicine (N.J.K.), University of Minnesota, Minneapolis, Minnesota; Department of Radiology (M.C.O.), Hennepin County Medical Center, Minneapolis, Minnesota; and Biostatistics Design and Analysis Center (L.Z.), Clinical and Translational Science Institute, University of Minnesota, Minneapolis, Minnesota. munosuppression for marrow transplantation, with a shared imaging appearance on DWI from multiple potential etiologies. ${ }^{4}$ The clinical features of ATL are quite variable but are abrupt in onset, ranging from minor cognitive impairment to severe neurologic dysfunction, depending on the extent of involved areas of the brain. ${ }^{1-3}$ Notably, while this damage is best initially depicted on DWI, with focal or symmetric reduced diffusion in the periventricular white matter (PVWM) with or without accompanying lesions on FLAIR, histopathology has noted both intramyelinic and oligodendroglial swelling. ${ }^{2-4}$ Recognizing this condi-

Please address correspondence to Can Özütemiz, MD, Department of Radiology, University of Minnesota MMC 292, 420 Delaware St, SE Minneapolis, MN 55455; e-mail: ozutemiz@umn.edu; @CanOzutemiz

- Indicates open access to non-subscribers at www.ajnr.org

$\equiv$ Indicates article with on-line appendix.

http://dx.doi.org/10.3174/ajnr.A5947 
tion is crucial because it is a clinicoradiologic diagnosis in which the symptoms and imaging findings may reverse abruptly upon removal of the underlying cause; alternatively, ATL can be fatal. ${ }^{2}$

Various toxic etiologies have been reported to cause ATL, including chemotherapeutic and immunosuppressive agents, substance abuse (particularly opiates), antimicrobials, environmental toxins, hepatic failure, and others. ${ }^{2,4-10}$ Notably, ATL has an overall worse prognosis than posterior reversible encephalopathy syndrome (PRES), another potentially reversible disorder that can also occur with chemotherapy or immunosuppression. ${ }^{4}$ However, it is unknown whether the outcomes from these various causes of ATL are similar and whether they have differences in initial imaging severity; it is plausible that severe imaging findings could portend worse outcome in particular settings, which would emphasize the importance of prompt recognition of ATL in particular clinical scenarios.

Given that prior studies were smaller and preliminary, the goal of this larger study was to evaluate various causes of ATL, to determine whether the imaging severity correlates with the clinical outcome, to evaluate differences in imaging severity and outcome among various etiologies/subgroups of ATL, and, secondarily, to describe any new causes of ATL.

\section{MATERIALS AND METHODS \\ Patients: Inclusion/Exclusion Criteria}

This retrospective, observational study was approved by the institutional review boards of University of Minnesota Medical Center and Hennepin County Medical Center (a quaternary care and a level 1 trauma center). Patients with suspected ATL were identified by PACS archives over 16 years (June 2002 to June 2018), as well as a radiological information system report search for the terms "leukoencephalopathy" or "toxic" in patients having undergone MR imaging, using 2 software programs: Vitrea Intelligence (Vital Images, Minnetonka, Minnesota) and Primordial communicator module of Powerscribe (Nuance Communications, Burlington, Massachusetts). These 2 methods yielded 172 patients suspected of having ATL on MR imaging before in-depth electronic health record review; thereafter, their electronic health records were reviewed by a quartet of a neuroradiology fellow, a radiology resident, a research fellow, and a medical student (C.O., J.C.B., S.K.R., N.J.K.) to assess whether patients were also clinically confirmed as having ATL or whether there was any positive clinical finding correlating with imaging such as withdrawal of the culprit agent and subsequent resolution of imaging/symptoms. The MR images were then reviewed separately by 2 staff neuroradiologists (A.M.M. and J.B.R. in University of Minnesota Medical Center and A.M.M. and M.C.O. in Hennepin County Medical Center, each with $>10$ years' experience) to include those with ATL on MR imaging. Because there are overlapping etiologies that may cause both ATL and PRES, the neuroradiologists ensured that there was no cortical edema, which has been described as the earliest manifestation of PRES; thus, patients were included only if they had reduced diffusion involving the white matter. ${ }^{11-13}$

Inclusion criteria for formal review (of imaging severity and outcome) included, at a minimum, the following: MR imaging within 3 weeks of presentation, including DWI with ADC maps, and FLAIR images obtained in the acute setting when patients were still symptomatic, either in the pretreatment or early treatment era, as well as MR imaging with reduced diffusion in the white matter suspicious for ATL. ${ }^{2}$ Patients were excluded if there was a lack of DWI or ADC maps, lack of measured ADC reduction, CTL or MR imaging findings not suggestive of ATL (eg, predominantly cortical edema), other PVWM disorders with reduced diffusion (eg, lymphoma, primary brain tumor, infarction, trauma); or they were younger than 10 years of age or had infectious disorders, hypoxic-ischemic insults, leukodystrophies, severely compromised MR imaging data, clinical findings not indicating ATL (eg, lack of acute neurologic symptoms, discordance between clinical and radiologic findings), and potential confounding conditions (eg, cranial metastasis or the presence of leptomeningeal enhancement) (Fig 1).

\section{MR Imaging Acquisition}

During a 15-year period, multiple $1.5 \mathrm{~T}$ and $3 \mathrm{~T}$ MR imaging units were used, so the sequence parameters varied. However, the general ranges were as follows for FLAIR, DWI, SWI, postcontrast T1WI, and MRA-FLAIR: TR/TE/TI/NEX/echo-train length at 1.5T: $6000-9000 \mathrm{~ms} / 105-110 \mathrm{~ms} / 2000-2200 \mathrm{~ms} / 1-2 / 15-23$; at 3T: $8000-11,000 \mathrm{~ms} / 100-120 \mathrm{~ms} / 2000-2200 \mathrm{~ms} / 1-2 / 11-25$; on 1.5T DWI: TR/TE, $3100-4000 \mathrm{~ms} / 70-120 \mathrm{~ms}$; on 3T: TR/TE, 2700-3000 ms/60-100 ms; gradient strength $b=1000 \mathrm{~s} / \mathrm{mm}^{2}$; SWI: TR/TE/NEX/flip angle at $1.5 \mathrm{~T}: 49 \mathrm{~ms} / 40 \mathrm{~ms} / 1 / 15^{\circ}$; at $3 \mathrm{~T}: 20$ $\mathrm{ms} / 27 \mathrm{~ms} / 1 / 15^{\circ}$; postcontrast T1WI: TR/TE/NEX/echo-train length at 1.5T: $420-500 \mathrm{~ms} / 12-15 \mathrm{~ms} / 2-3 / 35$; at $3 \mathrm{~T}$ : $500-650$ ms/11-15 ms/1-2/35; TOF-MRA: TR/TE/NEX at 1.5T, 35-40 $\mathrm{ms} / 7 \mathrm{~ms} / 1$; at $3 \mathrm{~T}, 20-25 \mathrm{~ms} / 3-4 \mathrm{~ms} / 1$. The slice thickness for FLAIR, DWI, and postcontrast T1WI was $5 \mathrm{~mm}$; for SWI, it was 2 $\mathrm{mm}$; and for TOF-MRA, it was $0.9 \mathrm{~mm}$ (4 slabs).

\section{MR Imaging Review, Grading of Radiologic Severity, and ADC Measurements}

ADC measurements were obtained from the lowest ADC value region and averaging 3 ROIs from these abnormal regions. ROI sizes were selected as either the smallest or the default size allowed by the PACS, varying between 0.165 and $0.371 \mathrm{~cm}^{2}$ for standardization of the measurements. If ATL was bilateral, the ROIs were averaged between sides; if unilateral, the 3 ROIs were averaged from that lesion. The percentage ADC reduction in visibly affected regions was calculated and compared with normal-appearing white matter, usually being the anterior frontal PVWM, by averaging $\geq 3$ ADC-value ROIs. All ADC measurements were obtained by a neuroradiology fellow (C.O.) separately, before MR imaging severity scoring.

The initial MR imaging severity of DWI and FLAIR was graded independently by 2 staff neuroradiologists using a previously described system, ranging from grades 0 to $4(0=$ normal, $4=$ severe; see the On-line Appendix for more information). ${ }^{2,14}$ In a few cases in which scoring was different, these images were evaluated jointly by the 2 neuroradiologists in a separate session. SWI and postcontrast T1WI were also assessed, as well as follow-up MR imaging, when available. CTA or MRA was also evaluated, if available, for any additional positive findings. 


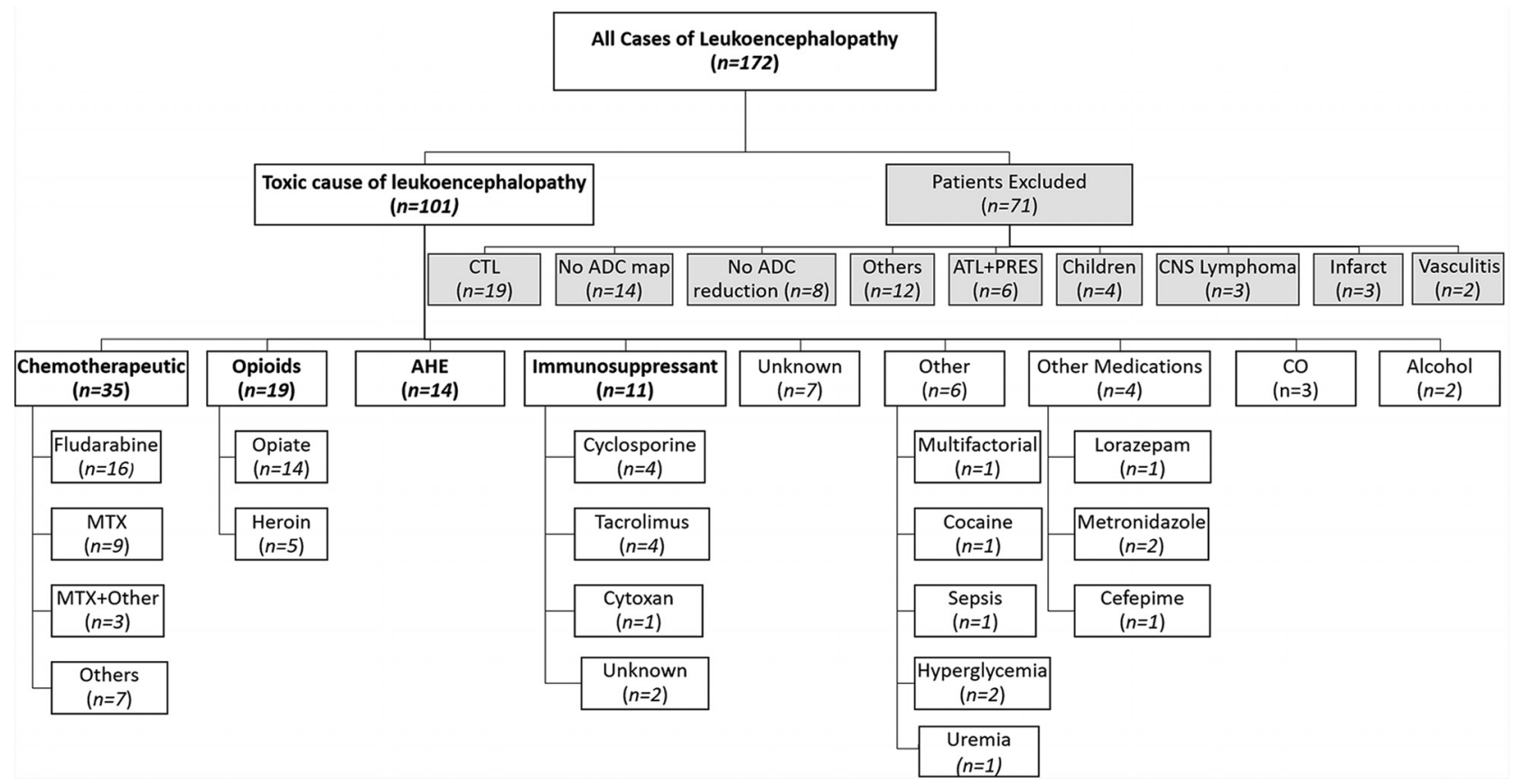

FIG 1. Organizational chart of inclusion of 101 patients with ATL in this study. MTX = Methotrexate; CO = carbon monoxide; CTL = Chronic toxic leukoencephalopathy; ATL+PRES = Acute toxic leukoencephalopathy with concomitant posterior reversible encephalopathy syndrome.

Table 1: Summary of variables with respect to groups of cause of ATL

\begin{tabular}{|c|c|c|c|c|c|c|c|c|c|c|c|}
\hline Variable & Statistic & $\begin{array}{l}\text { All ATL } \\
(n=101)\end{array}$ & $\begin{array}{c}\text { CTX } \\
(n=35)\end{array}$ & $\begin{array}{c}\mathrm{O} / \mathrm{H} \\
(n=19)\end{array}$ & $\begin{array}{c}\text { AHE } \\
(n=14)\end{array}$ & $\begin{array}{c}\text { ID } \\
(n=11)\end{array}$ & $\begin{array}{c}\text { Unknown } \\
(n=7)\end{array}$ & $\begin{array}{l}\text { Other } \\
(n=6)\end{array}$ & $\begin{array}{l}\mathrm{PM}^{\mathrm{a}} \\
(n=4)\end{array}$ & $\begin{array}{c}\mathrm{CO} \\
(n=3)\end{array}$ & $\begin{array}{c}\text { Alcohol } \\
(n=2)\end{array}$ \\
\hline \multirow[t]{2}{*}{ Sex } & Male & 50 & 17 & 11 & 7 & 2 & 2 & 4 & 2 & 3 & 2 \\
\hline & Female & 51 & 18 & 8 & 7 & 9 & 5 & 2 & 2 & 0 & 0 \\
\hline $\begin{array}{l}\text { Age } \\
\text { (years) }\end{array}$ & $\begin{array}{l}\text { Mean } \\
(\mathrm{SD})\end{array}$ & 40.72 (17.6) & $41.3(18.9)$ & 34.6 (14.7) & 44.6 (14.2) & $52.1(19.7)$ & $33.6(13.0)$ & $36.4(21.2)$ & $49.0(20-68)^{b}$ & $31.3(20-44)^{b}$ & $31.0(25-37)^{b}$ \\
\hline $\begin{array}{l}\text { mRS } \\
\text { score }\end{array}$ & $\begin{array}{l}\text { Mean } \\
\text { (SD) }\end{array}$ & $1.97(2.1)$ & $2.71(2.2)$ & $2.33(2.0)$ & $2.07(2.2)$ & $0.40(0.5)$ & $1.50(1.6)$ & $1.80(2.5)$ & $0.25(0-1)^{b}$ & $1.67(0-5)^{b}$ & $0.50(0-1)^{\mathrm{b}}$ \\
\hline $\begin{array}{r}\text { ATLOS } \\
\text { score }\end{array}$ & $\begin{array}{l}\text { Mean } \\
\text { (SD) }\end{array}$ & $1.60(1.6)$ & $2.29(1.7)$ & $1.67(1.4)$ & $1.57(1.7)$ & $0.60(0.97)$ & $1.17(1.2)$ & $1.40(2.0)$ & $0.25(0-1)^{b}$ & $1.33(0-4)^{b}$ & $0.50(0-1)^{b}$ \\
\hline $\begin{array}{l}\text { DWI } \\
\text { severity }\end{array}$ & $\begin{array}{c}\text { Mean } \\
\quad(S D)\end{array}$ & 2.59 (1.1) & $2.63(1.0)$ & $3.26(1.1)$ & $2.07(0.9)$ & $2.00(0.77)$ & 2.14 (1.2) & $3.20(1.3)$ & $2.75(2-4)^{b}$ & $2.67(1-4)^{b}$ & $2.00(1-3)^{b}$ \\
\hline $\begin{array}{l}\text { FLAIR } \\
\text { severity }\end{array}$ & $\begin{array}{l}\text { Mean } \\
\text { (SD) }\end{array}$ & $2.52(1.2)$ & $2.34(1.3)$ & $3.32(0.9)$ & $2.15(1.0)$ & $2.55(0.5)$ & $2.00(1.3)$ & $3.40(1.3)$ & $1.67(1-3)^{\mathrm{b}}$ & $2.33(0-4)^{b}$ & $1.50(1-2)^{\mathrm{b}}$ \\
\hline $\begin{array}{l}\text { ADC \% } \\
\text { loss }\end{array}$ & $\begin{array}{l}\text { Mean } \\
(\mathrm{SD})\end{array}$ & $\begin{array}{l}35.6 \% \\
\text { (17.9) }\end{array}$ & $\begin{array}{r}34.0 \% \\
(20.1)\end{array}$ & $\begin{array}{l}41.3 \% \\
(16.2)\end{array}$ & $\begin{array}{l}30.5 \% \\
\text { (14.2) }\end{array}$ & $\begin{array}{r}28.2 \% \\
(13.7)\end{array}$ & $\begin{array}{l}32.4 \% \\
(20.0)\end{array}$ & $\begin{array}{r}34.3 \% \\
\text { (13.7) }\end{array}$ & $\begin{array}{l}44.4 \% \\
(20.8-62.1)^{\mathrm{b}}\end{array}$ & $\begin{array}{l}63.8 \% \\
(58.9-71.6)^{b}\end{array}$ & $\begin{array}{l}38.9 \% \\
(29.5-48)^{b}\end{array}$ \\
\hline
\end{tabular}

Note:-CTX indicates Chemotherapeutics; O/H, Opiates/Heroin; AHE, Acute Hepatic Encephalopathy; ID, Immunosuppressive drugs; PM, Prescribed medications; CO, Carbon Monoxide.

${ }^{a}$ Including metronidazole $(n=2)$, lorazepam $(n=1)$, and cefepime $(n=1)$.

${ }^{\mathrm{b}}$ Minimum-Maximum.

\section{Clinical Outcome Scoring}

To score the clinical outcome, 2 scoring methods were used: the mRS (based on a clinical follow-up time at $>90$ days) and a previously published ATL outcome score (ATLOS, based on clinical follow-up of $>30$ days). ${ }^{2,15}$ During the electronic health record review, the etiology of ATL was explained if clearly identified. Regarding laboratory tests, results of a basic metabolic panel and CSF tests obtained within a time window of 1 week before and after the initial brain MR imaging were evaluated if available and clinically important abnormal values were recorded.

\section{Statistical Evaluation}

A biostatistician performed the calculations. For statistical comparison among subgroups, subcategories of ATL etiologies were compared consisting of $>6$ patients; these subgroups were compared using the Kruskal-Wallis (continuous variables), Fisher exact (categoric variables), and pair-wise comparison (subgroupsubgroup) tests, to evaluate significant differences in MR imaging severity or outcomes among subgroups. For pair-wise comparisons, Kruskal-Wallis (with 2 groups) tests were used. The Wilcoxon test was used to compare MR imaging measures of the subjects with the most severe clinical outcomes, with those with complete symptom resolution. To adjust for multiple pair-wise comparisons, the $P$ value threshold for significance was set to $P<.0083$ using Bonferroni's method. Spearman correlations were then obtained separately between each of the following variables, including age, FLAIR MRI severity, DWI MRI severity, mRS and ATLOS outcome scores, while the Wilcoxon test was 


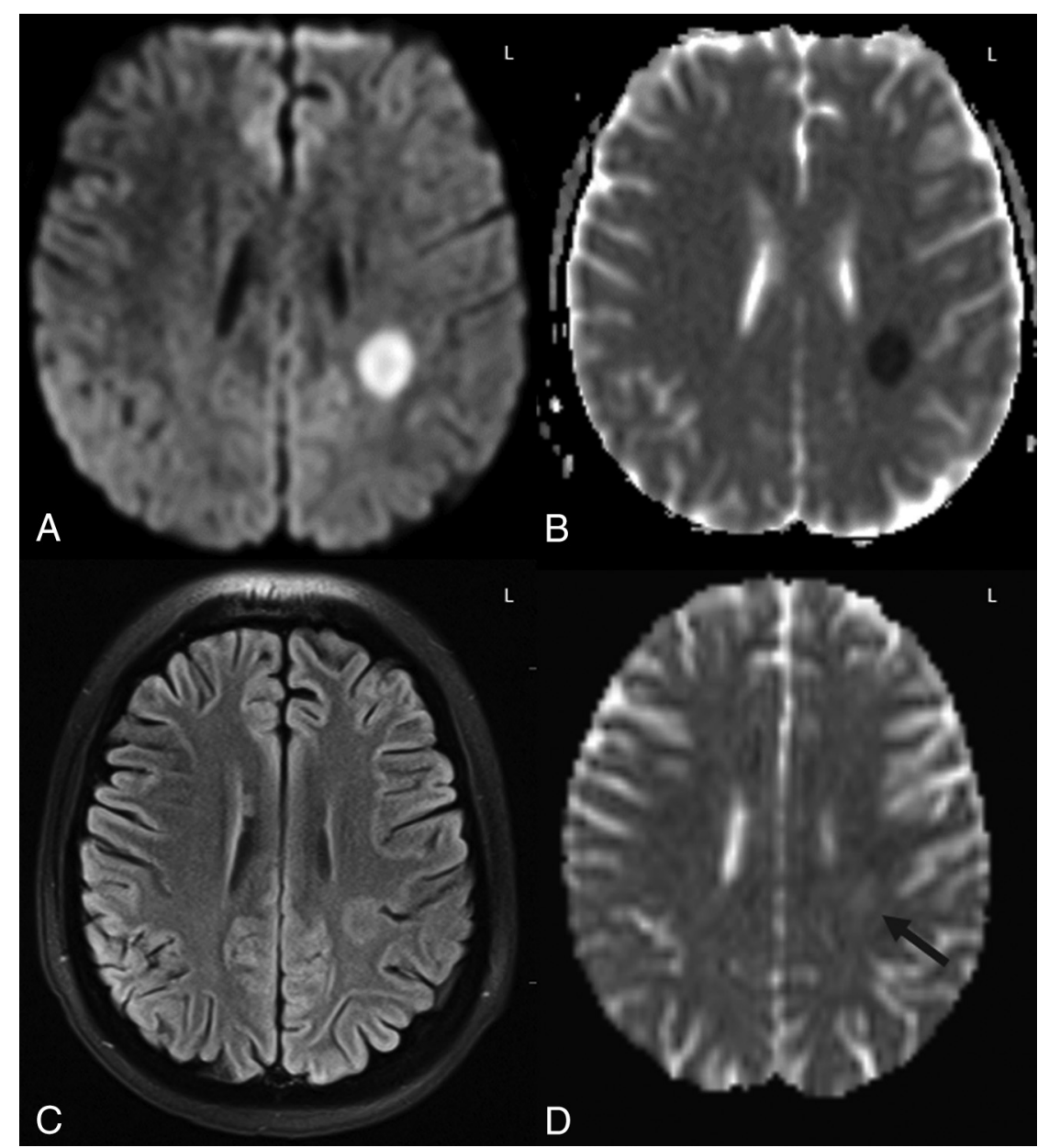

FIG 2. Example of grade 1 DWI-FLAIR ATL severity. A 16-year-old girl with acute leukemia after 5 days of high-dose cytarabine and clofarabine combined chemotherapy, presented with acute right upper extremity weakness. An initial head CT (not shown) was unremarkable. On DWI ( $A$ ) and ADC (B), unilateral diffusion reduction was present in the left PVWM with $60 \%$ ADC loss compared with NAWM and corresponding faint FLAIR hyperintensity (C). Accompanying MRA study (not shown) was normal. D, On follow-up MR imaging at 2 weeks after withdrawal of the chemotherapy, there was normalization of diffusion reduction with faint residual hyperintensity on DWI (not shown), ADC map (D, black arrow), and FLAIR (not shown), with a resultant T2-shinethrough effect on DWI. The symptoms had resolved at long-term clinical follow-up with both the ATLOS and $\mathrm{mRS}$ clinical scores being 0 .

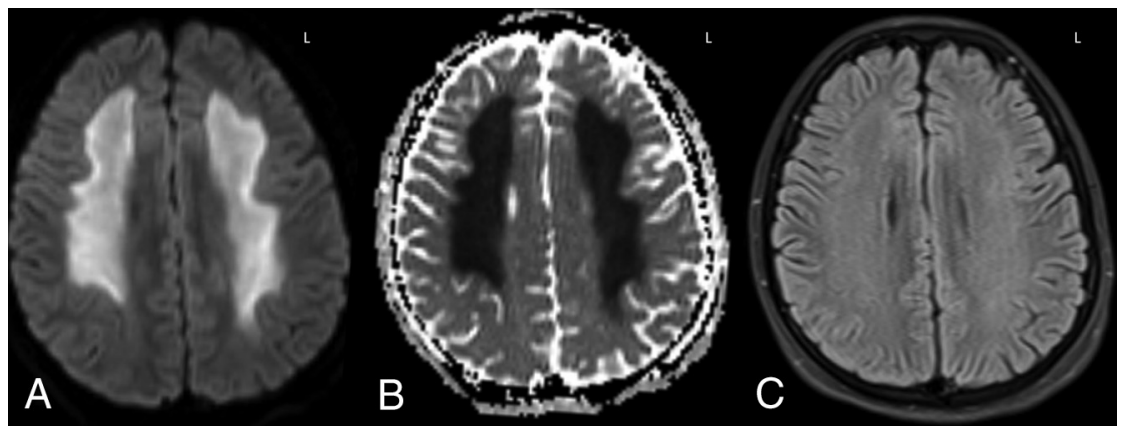

FIG 3. Example of a grade 2 DWI-FLAIR ATL severity from uremia. A previously healthy 16 -yearold boy with coma had renal failure of unknown cause, blood urea nitrogen level of $>115 \mathrm{mg} / \mathrm{dL}$ (normal $<25 \mathrm{mg} / \mathrm{dL}$ ), creatinine level of $18.34 \mathrm{mg} / \mathrm{dL}$ (normal, $<1.2 \mathrm{mg} / \mathrm{dL}$ ), glomerular filtration rate of 5 (normal, >90), and systolic blood pressure of $>160 \mathrm{~mm} \mathrm{Hg}$. MR imaging revealed PVWM abnormalities sparing the basal ganglia on DWI ( $A$ and $B)$, with a normal appearance on FLAIR $(C)$ and normal MRA (not shown). Laboratory work-up only revealed uremia. Clinically, the symptoms resolved with dialysis, and MR imaging 2 days later (not shown) noted continued diffusion reduction, while the ADC values rose with complete resolution of symptoms (the ATLOS and mRS scores were 0 ). used to evaluate for differences between gender $(P<.05$ used for both the Spearman and Wilcoxon tests). All analyses were obtained using SAS (Version 9.4; SAS Institute, Cary, North Carolina).

\section{RESULTS}

\section{Patient Demographics and}

\section{Subcategories of ATL}

Ultimately, 101 patients with ATL were included, with 71 excluded, most commonly due to CTL $(n=19)$, as shown in Fig 1. The subcategories of causes with $n>6$ were chemotherapy $(n=35)$, opiates $(n=19)$, acute hepatic encephalopathy (AHE, $n=14$ ), and immunosuppressive drugs $(n=11)$, as shown in Table 1. There were no significant differences in sex or age among each of the 4 subgroups of $>6$ (each, $P>.05$ ). Samples of patients' MR images are depicted in Figs 2-5.

\section{MR Imaging Severity Scoring and Subgroup Comparisons}

Table 1 depicts the mean severities on DWI and FLAIR of all patients. There were significant differences overall between the mean DWI $(P=.003)$ and FLAIR $(P=.009)$ MR imaging severity scores among the 4 major subgroups. Notably, opiate abuse had the highest mean severity on both sequences, while patients who were immunosuppressed and those with AHE had the least mean severity on DWI and FLAIR, respectively. Pair-wise intergroup comparisons of the MR imaging severities and outcomes of the 4 subgroups are provided in Table 2. Notably, 6/101 patients with ATL had only reversible splenial lesions (grade 1 severity) with complete symptom resolution; of these, the causes in 4 different patients were the following: chemotherapy, immunosuppressive medication, sepsis, and alcohol toxicity, while in the remaining 2 patients, the etiology was unknown.

\section{Clinical Outcome Severities and Subgroup Comparisons}

Table 1 provides the mean clinical outcome score on mRS at $>90$ days and on ATLOS at $>30$ days for each subgroup; in 7 patients, outcomes could not be discerned. Overall, significant differences were found among the 4 major sub- 


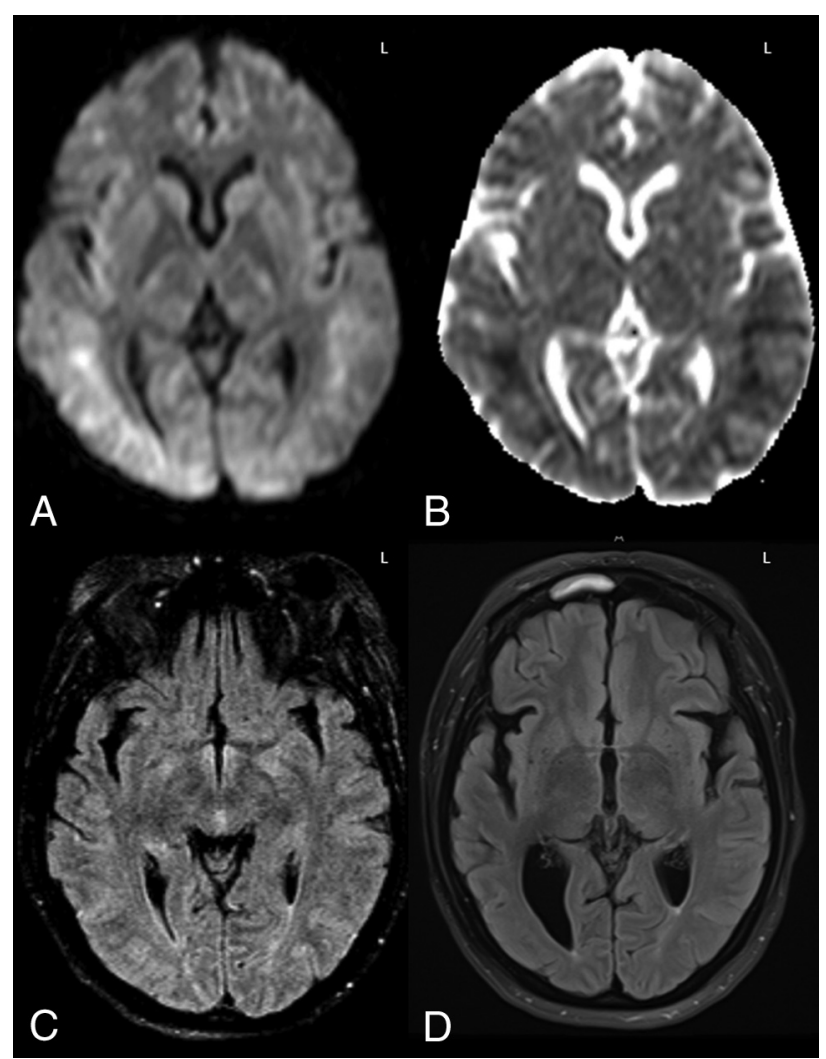

FIG 4. A 40-year-old woman with altered mentation 1 month postliver transplantation, who was immunosuppressed with tacrolimus. MR imaging demonstrated extensive diffusion reduction bilaterally in the PVWM and subcortical white matter on DWI and ADC ( $A$ and $B)$, with $58 \%$ ADC loss (versus NAWM) and slight hyperintensity on FLAIR (C) involving 3 lobes, consistent with grade 3 ATL imaging severity on DWI and FLAIR. Note that there was no apparent cortical edema on FLAIR to suggest PRES. After the withdrawal of tacrolimus and supportive therapy, the long-term ATLOS and mRS scores were both 0 . $D$, An MR imaging 12 years later (obtained for chronic headaches) showed mild-moderate right PVWM loss and ex-vacuo dilation of the occipital horn of the right lateral ventricle as sequelae of ATL, without cortical encephalomalacia.

groups for both the mRS $(P=.025)$ and ATLOS $(P=.032)$ scores. When we compared these subgroup outcomes against each other (Table 2), there were significant differences between the chemotherapy-versus-immunosuppressed subgroups for both scores, and between opiate-versus-immunosuppressed groups based on the mRS only; the other intergroup comparisons did not have significant differences (each, $P>.0083$ ).

Severe outcomes (grade 4 on ATLOS or grade 5-6 on mRS) were noted in 22/94 (23.4\%) patients with available data in this study, 16 of whom died and 6 being comatose at 90 days postpresentation; 13 of these 22 patients had severe outcomes from chemotherapy. In the 16 patients who died, the etiologies were the following: fludarabine $(n=9)$, AHE $(n=3)$, and 1 each of methotrexate, opiates, hyperglycemia, and rituximab. In the 16 patients who died, the cause of death was often other comorbidities such as sepsis, organ failure, cardiac arrest, or diffuse malignancy, though their neurologic symptoms related to ATL never subsided. At the other end of the clinical outcome spectrum, there was symptom resolution (ATLOS or mRS grade 0) in 34/94 (36.1\%) patients with ATL, with 26/94 (27.6\%) having only minimal se-

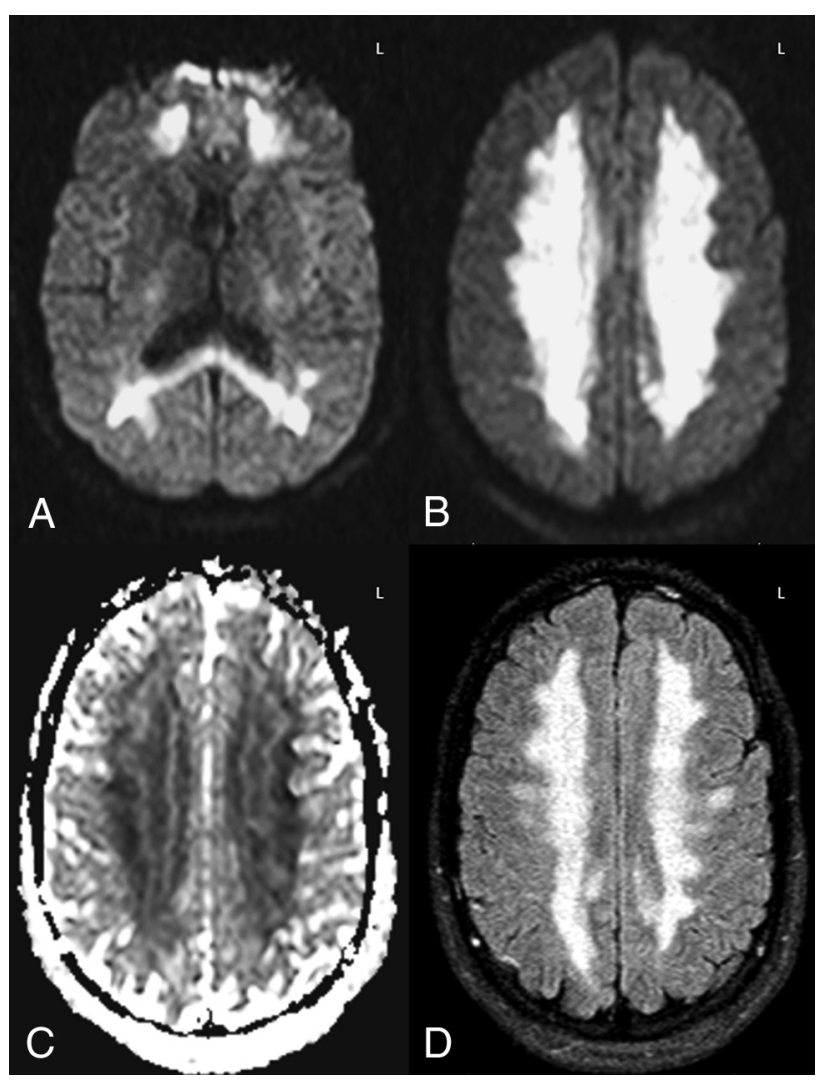

FIG 5. Example of opiate-related grade 4 DWI-FLAIR ATL severity. A 26-year-old comatose man with urine toxicology positive for propoxyphene (an opioid analgesic) had diffuse and confluent DWIADC $(A-C)$ and FLAIR $(D)$ abnormalities with $67 \%$ ADC loss versus NAWM. The patient had moderate long-term neurologic sequelae, with the ATLOS at 1 month being 2 and the mRS at 3 months being 3 . Follow-up MR imaging was not performed.

quelae (ATLOS grade 1 or mRS grades 1-2). When we compared the subjects with the most severe clinical outcomes $(n=22)$ with those with complete symptom resolution $(n=34)$ regardless of different etiologies, DWI and FLAIR imaging scores were higher in the severe-outcomes group compared with the symptomresolution group. DWI $\mathrm{D}_{\text {Mean }}$ was 3.1 versus $2.2(P=.002)$, and FLAIR $_{\text {mean }}$ was 3 versus $2(P=.005)$, respectively.

\section{Correlation of MR Imaging Severity of Subgroups with Outcomes}

There was a strong correlation between DWI and FLAIR severity $(\rho=0.731, P<.0001)$ and between the mRS and ATLOS scores $(\rho=$ $0.978, P<.0001)$. There were mild-moderate correlations between both the DWI and FLAIR severity with both the mRS and ATLOS ( $\rho=0.289-.349, P \leq .005$ ) as shown in Table 3. Regarding the 4 subcategories of $>6$, both DWI and FLAIR severity mildly to moderately correlated with the outcome scores for opiates $(\rho=0.472$ $0.608, P<.05)$ and chemotherapy $(\rho=0.460-0.586, P<.05)$. There was no significant correlation of age or sex with either DWI or FLAIR severity or with either clinical outcome score (each, $P>.005$ ).

\section{ADC Measurements and Other MR Imaging/MRA Findings}

A boxplot (Fig 6) shows that the greatest percentage ADC reduction was noted in the affected PVWM of carbon monoxide toxicity

AJNR Am J Neuroradiol 40:267-75 Feb 2019 www.ajnr.org

271 
Table 2: Pair-wise intergroup comparison of clinical outcomes among the 4 largest subgroups causing ATL

\begin{tabular}{|c|c|c|c|c|c|c|}
\hline & \multicolumn{6}{|c|}{$P$ Values for Pair-wise Comparison } \\
\hline & $\begin{array}{l}\text { Chemotherapy vs } \\
\text { Immunosuppressant }\end{array}$ & $\begin{array}{l}\text { Chemotherapy } \\
\text { vs AHE }\end{array}$ & $\begin{array}{l}\text { Chemotherapy } \\
\text { vs Opiates }\end{array}$ & $\begin{array}{c}\text { Immunosuppressant } \\
\text { vs AHE }\end{array}$ & $\begin{array}{l}\text { Immunosuppressant } \\
\text { vs Opiates }\end{array}$ & $\begin{array}{l}\text { Opiates } \\
\text { vs AHE }\end{array}$ \\
\hline $\mathrm{mRS}$ & $.004^{b}$ & .32 & .62 & .07 & $.007^{b}$ & .61 \\
\hline ATLOS & $.006^{\mathrm{b}}$ & .16 & .23 & .19 & .031 & .66 \\
\hline DWI severity & .08 & .08 & .03 & .93 & $.003^{b}$ & $.003^{b}$ \\
\hline FLAIR severity & .90 & .50 & $.006^{\mathrm{b}}$ & .25 & .015 & $.003^{b}$ \\
\hline
\end{tabular}

a Multiple comparisons were adjusted with a Bonferroni correction $(P=.0083)$.

b Significant.

Table 3: Correlation between MRI severity and clinical outcome severity

\begin{tabular}{|c|c|c|c|c|c|c|c|c|c|c|}
\hline & \multicolumn{2}{|c|}{ All Patients } & \multicolumn{2}{|c|}{ Chemotherapeutics } & \multicolumn{2}{|c|}{ Opiates } & \multicolumn{2}{|c|}{ AHE } & \multicolumn{2}{|c|}{ Immunosuppressants } \\
\hline & $\mathrm{mRS}$ & ATLOS & $\mathrm{mRS}$ & ATLOS & mRS & ATLOS & mRS & ATLOS & $\mathrm{mRS}$ & ATLOS \\
\hline \multicolumn{11}{|c|}{ DWI severity } \\
\hline$\rho$ & .349 & .289 & .478 & .460 & .608 & .573 & -.123 & -.240 & .000 & -.103 \\
\hline$P$ & $.001^{\mathrm{a}}$ & $.005^{\mathrm{a}}$ & $.006^{\mathrm{a}}$ & $.009^{\mathrm{a}}$ & $.007^{\mathrm{a}}$ & $.012^{\mathrm{a}}$ & .674 & .408 & 1.00 & .777 \\
\hline \multicolumn{11}{|c|}{ FLAIR severity } \\
\hline$\rho$ & .336 & .332 & .543 & .586 & .472 & .412 & .123 & .135 & .000 & .079 \\
\hline$P$ & $.001^{\mathrm{a}}$ & $.001^{\mathrm{a}}$ & $.001^{\mathrm{a}}$ & $.001^{\mathrm{a}}$ & $.047^{\mathrm{a}}$ & .088 & .689 & .659 & 1.00 & .826 \\
\hline
\end{tabular}

${ }^{a}$ Significant.

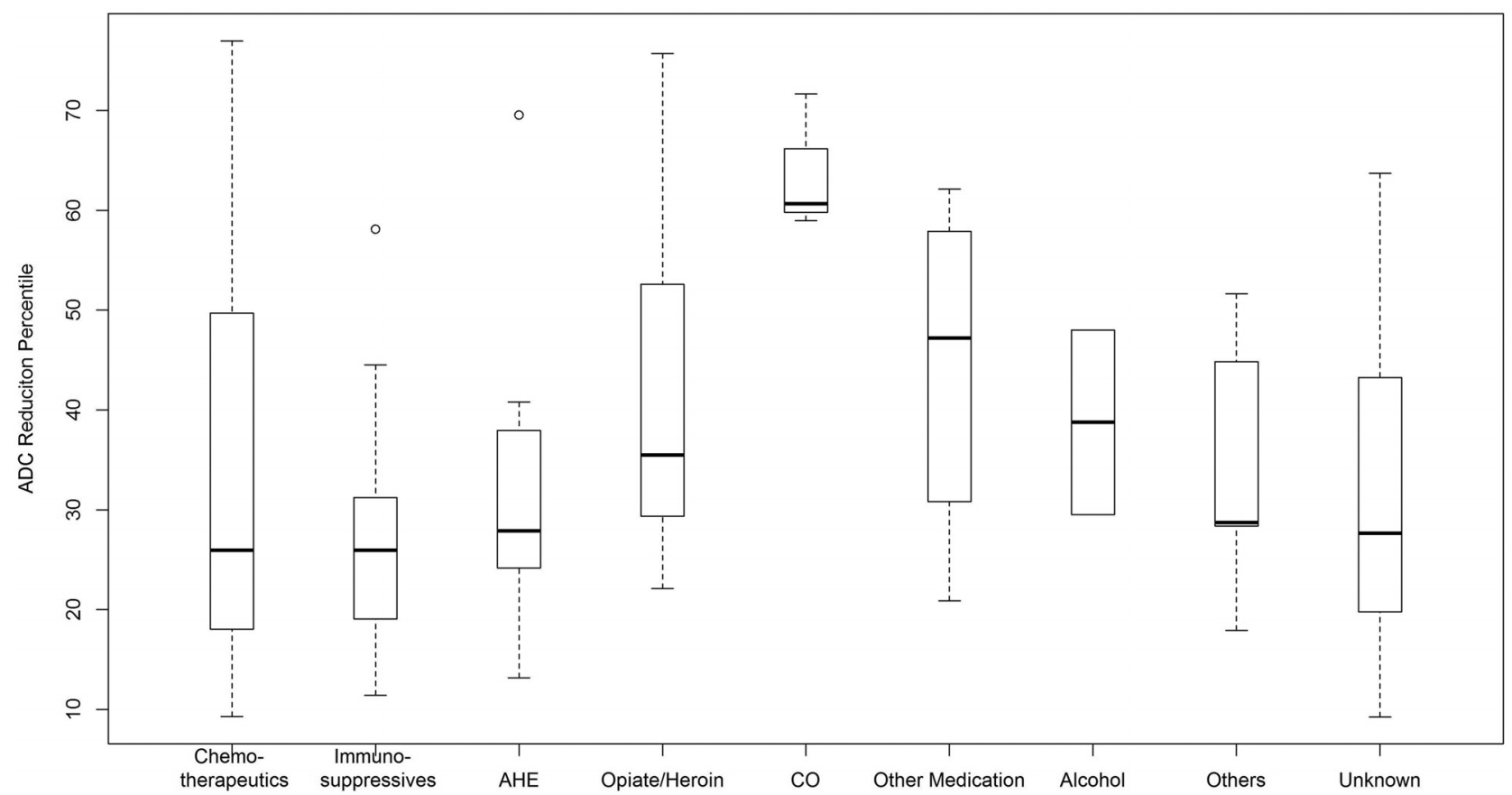

FIG 6. Boxplot of each etiologic subgroup with the percentage ADC reduction of affected regions compared with NAWM. ATL from carbon monoxide (CO, a small subgroup of 3 ) had a much greater percentage ADC reduction versus the other subgroups.

(mean, 64.0\%), while the least was the immunosuppressive medication subgroup (mean, 28.2\%). Regarding other MR imaging findings, contrast-enhanced T1WI was available in 72 patients, without any $>1-\mathrm{cm}$-size enhancing foci, though punctate foci were found in $7 / 72(9.0 \%)$ amid the PVWM lesions on DWI. SWI was available in 52 patients (T2*WI, in 8 others), with punctate microhemorrhages in $9 / 60$ patients (15\%); 5 of these 9 had an $m R S \leq 1$. MRA was available in 23/101 patients, and the findings of only 1 were positive (an incidental aneurysm).

\section{Additional Laboratory Findings}

Of 101 patients with ATL, 45 had a lumbar puncture; 24/45 had positive CSF findings. Most notably, in the 9 patients with an available myelin basic protein (MBP) level, 8 had increased MBP (4 from fludarabines, 3 from opiates, 1 unknown), 9 had increased total protein, 9 had increased glucose, and 2 had findings positive for oligoclonal bands. A metabolic panel was available in 91 patients; 54/91 had a positive finding-most notably, the blood urea nitrogen level was increased in 24 of these 91 patients.

\section{DISCUSSION}

ATL is an uncommon condition that should be considered in the differential diagnosis of patients with acute encephalopathy. It is a clinicoradiologic diagnosis characterized by cerebral WM injury on DWI from a toxic substance and should be distinguished from 
other clinical disorders such as PRES, hypoxic-ischemic encephalopathy, meningitis, encephalitis, tumor recurrence, metastasis, stroke, acute disseminated encephalomyelitis, and other metabolic syndromes. Recognizing this uncommon disorder is vital because it can be potentially reversible with prompt removal of the underlying cause and supportive therapy, while the clinical outcome varies among etiologies. Given the shared imaging appearance on DWI of the various causes of ATL, it is important to both diagnose this condition and assist the ordering physician in determining an underlying etiology, given the differences in outcomes among causes. However, to our knowledge, no larger study has as yet evaluated differences in outcomes among different etiologies of ATL.

Many etiologies have been reported for ATL, including a variety of chemotherapeutic and immunosuppressive medications, opiate ingestion (whether prescribed or illicit), some antimicrobial medications, various metabolic disorders (such as hyperammonemia from AHE or hyperglycemia), carbon monoxide poisoning, and, recently, even a commercially available dietary supplement. ${ }^{2-10,16-18}$ In this study, chemotherapy was the most common cause, followed by opioids, AHE, and immunosuppressants, while the cause was unknown in $7 \%$. According to these results, each cause may affect each age group and sex equally, but the imaging severity and clinical outcomes differ among the types of etiologies.

The exact pathophysiology of ATL is also unknown, but the evidence shows that white matter damage may occur directly from toxic injury on the myelin sheath or indirectly from capillary endothelial injury or a combination of both. ${ }^{2-4,13}$ For example, carbon monoxide and inhaled opiates may cause either toxic demyelination or spongiform degeneration of the PVWM. ${ }^{5,19}$ While chemotherapeutics such as fludarabine and methotrexate can cause direct toxic damage of the axons and myelin sheath, chemotherapeutic agents, such as methotrexate and 5-flourouracil, and immunosuppressive agents, such as tacrolimus and cyclosporine, were shown to disrupt the cerebral microvasculature. ${ }^{4,20-24} \mathrm{Re}$ gardless of the culprit agent, diffusion reduction in the PVWM may appear due to pathologic changes in the myelin from intramyelinic edema or toxic demyelination. , $^{2,3,5,8}$

The conditions of about one-third of the patients with ATL in this study were from chemotherapy, with fludarabine (16\%) being most common and methotrexate in $12 \%$. Fludarabine was also the most common cause of death from ATL (9/16 patients with ATL who died), in accord with prior literature that showed that fludarabine can cause severe, late-onset ATL (weeks after chemotherapy) and that ATL from fludarabine has worse outcomes relative to PRES from fludarabine. ${ }^{4,25}$ Thus, fludarabine likely accounts for the chemotherapy-related subgroup having the worst outcome.

Opioids were the second most common cause of ATL in this study (18.8\%), having the second worst outcomes of the 4 ATL subgroups that consisted of $>6$ patients, while this group tends to have the most severe extent on DWI. Opioids are lipophilic substances that can cross through the BBB and accumulate in fat-rich myelin when in high doses, thus causing cerebral or cerebellar symmetric spongiform degeneration. ${ }^{19,26}$ Particularly in the case of "chasing the dragon," the inhaled substance bypasses other organs, thus allowing faster, direct passage to the brain relative to the intravenous route. In addition, potentially superimposed hypoxia may induce BBB disruption, thus aggravating the toxic effects. ${ }^{27}$ Notably, opiate toxicity/overdose may present with several imaging patterns (such as basal ganglia injury), perhaps due to anoxic injury; hence, because this study focused solely on the PVWM pattern of injury of ATL, these results may not be extrapolated onto other imaging patterns.

AHE (13.8\%) was the third most common cause of ATL. Notably, AHE most commonly involves the insula or thalami, with variable PVWM involvement. ${ }^{9}$ Thus, this study included only AHE with solely PVWM lesions, which occurs in 15\% of the cases of AHE. ${ }^{9}$ The outcomes of AHE-related ATL in this study tended to be slightly better than those of chemotherapy- and opiate-related ATL.

The fourth largest subgroup was immunosuppressant medications $(10.9 \%)$. With the increasing implementation of marrow transplantation, ATL from immunosuppressants may be increasing in frequency in a fashion similar to that of another potentially reversible disorder, PRES. ${ }^{11,12,14,28}$ Thus, immunosuppressantrelated ATL had the best outcomes relative to the other subgroups of known causes in this study. However, both this study and prior ones have shown that ATL from immunosuppressive medications such as cyclosporine is still relatively uncommon. ${ }^{4,12}$

Another subgroup of ATL was carbon monoxide toxicity $(n=$ 3 ), which has at least 3 previously described patterns of involvement on DWI: PVWM, basal ganglia, or hippocampal (least common). ${ }^{5,29}$ Concomitant anoxic injury causing such lesions may also occur, but the PVWM variant was present in only a few patients in this study $(n<6)$, thus preventing a correlation of the clinical outcomes to the imaging severity.

Regarding the intergroup comparisons of imaging severity, the DWI severity was worse in the opiate subgroup versus the AHE and immunosuppressant subgroups, where ATL from opiates and chemotherapy tended to have more diffuse WM involvement. Meanwhile, ATL from AHE and immunosuppressants was milder and more focal. In most patients with ATL, the FLAIR lesions were less evident than on DWI or developed later, as shown previously. ${ }^{2}$

Regarding other imaging findings, contrast enhancement is not a common feature in ATL, being punctate and observed in only $9 \%$ of patients in this study, with differing outcomes; thus, the presence of enhancement likely neither changes the diagnosis nor affects outcome. Similarly, the presence of microhemorrhages (in 15\%) does not correlate with outcome and likely does not have clinical significance but may be related to underlying vasculopathy. ${ }^{30}$ MRA findings were largely negative, and only 1 patient had an incidental aneurysm.

Regarding the clinical outcomes from ATL and comparisons between subgroups, chemotherapy- and opiate-related ATL had significantly worse outcomes, despite the potentially reversible nature of ATL. Again, fludarabine likely accounts for the chemotherapy subgroup having the worst outcomes overall. In comparison, immunosuppressant-related ATL was less common in our population, with milder imaging severity and better outcomes, which is of note because one might expect that the comorbidities in immunosuppressed patients would precipitate worse out- 
comes. Although chemotherapy- and opiate-related ATL has worse clinical outcome scores compared with immunosuppressant-related ATL in pair-wise comparison (Table 2), only the opiate subgroup showed significant differences regarding imaging severity compared with the immunosuppressant group, which also supports the opiate-related ATL having the most severe and extensive DWI findings.

Regarding the AHE and immunosuppressant subgroups, the severity of imaging findings and clinical outcome did not significantly correlate in either of these 2 subgroups; this finding likely confirms the potentially reversible nature of ATL within these 2 subgroups. On the other hand, the opiate and chemotherapy subgroups of ATL had overall more severe MR imaging scores/extent, with moderate correlations between the imaging severity and outcome, suggesting that more diffuse brain involvement is likely to result in neurologic sequelae. Regardless of different etiologies, when the extent of abnormal DWI and FLAIR findings reached grade 3 or 4 (On-line Appendix), there was a higher possibility of severe outcomes.

The review of the laboratory values yielded interesting findings of MBP and blood urea nitrogen levels that may further elucidate the underlying pathophysiology of ATL. MBP is an important component of CNS myelin formation, and though its presence in CSF is nonspecific, increased levels of CSF-MBP suggest active demyelination in some syndromes, such as multiple sclerosis. $^{31,32}$ In this regard, a prior study focused on transplant neurotoxicity may have been the first of fludarabine-related ATL (having 4 patients from this study's cohort), and described high CSF-MBP levels. ${ }^{4}$ In addition, the current study may be the first description of elevated CSF-MBP in opiate-related ATL (3 patients) in humans, suggesting myelin damage; notably, CNS MBP is reduced with chronic demyelination in mice chronically exposed to opiates. ${ }^{33}$ Hence, CSF-MBP could be a potential marker to support the diagnosis of and evaluate the extent of myelin damage in ATL.

Another value, serum blood urea nitrogen, was increased in $26 \%$ of the patients with available values, secondary to either impaired kidney function or tumor lysis in patients in oncology. Most interesting, uremia has been described in association with PRES, whereas uremic encephalopathy may present on MR imaging with symmetric T2 hyperintensity of the basal ganglia. ${ }^{34}$ To our knowledge, the patient with uremia-related ATL described herein with PVWM involvement but sparing the basal ganglia may be the second such patient with uremia described with that imaging appearance (Fig 3). ${ }^{35}$ Furthermore, a recent study focused on concomitant ATL and PRES in a small cohort (a subset of patients excluded from this study) in which most had kidney failure and perhaps uremia. ${ }^{13}$ It is not known how uremia causes toxic leukoencephalopathy; however, diffusion tensor imaging studies found premature PVWM disease, especially in the anterior PVWM in patients with chronic kidney disease, and various uremic toxins may cause both endothelial dysfunction and cognitive impairment, especially via neuroexcitatory uremic-guanidine compounds. ${ }^{36,37}$ Given the number of patients with uremia in this study, it is plausible that uremia may exacerbate ATL or even predispose to ATL in the absence of other toxins.

There are several limitations to this study, the predominant one being that it was retrospective; for example, the clinical outcomes were based on retrospective evaluation of the patients' records. In addition, many patients, particularly within the chemotherapeutic and immunosuppressant groups, had multiple confounding factors that could affect the clinical outcome. Also, in the case of exposure to multiple potential toxic agents or medications, the MR imaging findings could vary due to superimposed nontoxic etiologies such as anoxia, sepsis, or renal failure. However, the authors note that this study focused on the outcomes from a common, shared imaging appearance of ATL involving the PVWM and included patients on that basis, not on the basis of etiology. Finally, multiple MRIs from different machines with $1.5 \mathrm{~T}$ and $3 \mathrm{~T}$ strengths were performed, whereas the differences in appearances and ADC values between $1.5 \mathrm{~T}$ and $3 \mathrm{~T}$ were not assessed in this study.

\section{CONCLUSIONS}

ATL is an imaging appearance that can arise from various etiologies, with potentially reversible reduced diffusion predominantly affecting the PVWM. Given the shared DWI appearance among this heterogeneous array of etiologies, their outcomes may differ. Thus, the neurologic symptoms completely resolved in $36 \%$, while severe outcomes occurred in $23 \%$. The clinical outcome was most severe with chemotherapy-related ATL (mostly due to fludarabine), whereas opiate toxicity had the second worst outcome and tends to have more severe extent on DWI. In contrast, immunosuppressive-related ATL tends to be more focal and clinically milder. These observations point to a need to study other clinical factors or comorbidities that influence the outcomes of ATL, such as the presence of uremia.

Disclosures: Mark C. Oswood-UNRELATED: Payment for Lectures Including Service on Speakers Bureaus: Philips Healthcare, Comments: sponsored lectures and speakers bureau in 2016-2017. Alexander M. McKinney-UNRELATED: Board Membership: VEEV, Inc., Minneapolis, MN, Comments: president and owner, informatics company; Patents (Planned, Pending or Issued): informatics patents; Royalties: Springer Nature, Chaum, Switzerland, Comments: book royalties.

\section{REFERENCES}

1. Filley CM, Kleinschmidt-DeMasters BK. Toxic leukoencephalopathy. N Engl J Med 2001;345:425-32 CrossRef Medline

2. McKinney AM, Kieffer SA, Paylor RT, et al. Acute toxic leukoencephalopathy: potential for reversibility clinically and on MRI with diffusion-weighted and FLAIR imaging. AJR Am J Roentgenol 2009;193:192-206 CrossRef Medline

3. Rimkus Cde M, Andrade CS, Leite Cda C, et al. Toxic leukoencephalopathies, including drug, medication, environmental, and radiation-induced encephalopathic syndromes. Semin Ultrasound CT MR 2014;35:97-117 CrossRef Medline

4. Beitinjaneh A, McKinney AM, Cao Q, et al. Toxic leukoencephalopathy following fludarabine-associated hematopoietic cell transplantation. Biol Blood Marrow Transplant 2011;17:300-08 CrossRef Medline

5. Teksam M, Casey SO, Michel E, et al. Diffusion-weighted MR imaging findings in carbon monoxide poisoning. Neuroradiology 2002; 44:109-13 CrossRef Medline

6. Keogh CF, Andrews GT, Spacey SD, et al. Neuroimaging features of heroin inhalation toxicity: "chasing the dragon." AJR Am J Roentgenol 2003;180:847-50 CrossRef Medline

7. Sandoval C, Kutscher M, Jayabose S, et al. Neurotoxicity of intrathecal methotrexate: MR imaging findings. AJNR Am J Neuroradiol 2003;24:1887-90 Medline 
8. Lucato LT, McKinney AM, Short J, et al. Reversible findings of restricted diffusion in 5-fluorouracil neurotoxicity. Australas Radiol 2006;50:364-68 CrossRef Medline

9. McKinney AM, Lohman BD, Sarikaya B, et al. Acute hepatic encephalopathy: diffusion-weighted and fluid-attenuated inversion recovery findings, and correlation with plasma ammonia level and clinical outcome. AJNR Am J Neuroradiol 2010;31:1471-79 CrossRef Medline

10. Kim E, Na DG, Kim EY, et al. MR imaging of metronidazole-induced encephalopathy: lesion distribution and diffusion-weighted imaging findings. AJNR Am J Neuroradiol 2007;28:1652-58 CrossRef Medline

11. Casey SO, Sampaio RC, Michel E, et al. Posterior reversible encephalopathy syndrome: utility of fluid-attenuated inversion recovery MR imaging in the detection of cortical and subcortical lesions. AJNR Am J Neuroradiol 2000;21:1199-206 Medline

12. McKinney AM, Short J, Truwit CL, et al. Posterior reversible encephalopathy syndrome: incidence of atypical regions of involvement and imaging findings. AJR Am J Roentgenol 2007;189:904-12 CrossRef Medline

13. Özütemiz C, Roshan SK, Kroll NJ, et al. Concomitant acute toxic leukoencephalopathy and posterior reversible encephalopathy syndrome. J Neuroimaging 2018;28:535-41 CrossRef Medline

14. Karia SJ, Rykken JB, McKinney ZJ, et al. Utility and significance of gadolinium-based contrast enhancement in posterior reversible encephalopathy syndrome. AJNR Am J Neuroradiol 2016;37:415-22 CrossRef Medline

15. Banks JL, Marotta CA. Outcomes validity and reliability of the modified Rankin Scale: implications for stroke clinical trials-a literature review and synthesis. Stroke 2007;38:1091-96 CrossRef Medline

16. Bartlett E, Mikulis DJ. Chasing "chasing the dragon" with MRI: leukoencephalopathy in drug abuse. Br J Radiol 2005;78:997-1004 CrossRef Medline

17. Schuuring J, Wesseling P, Verrips A. Severe tacrolimus leukoencephalopathy after liver transplantation. AJNR Am J Neuroradiol 2003;24:2085-88 Medline

18. Escamilla-Ocañas CE, Cámara-Lemarroy CR, Cantú-Martinez L, et al. Acute toxic leukoencephalopathy associated with a non-prescription weight loss supplement: a report of two cases. Neurol Sci 2017;38:2199-201 CrossRef Medline

19. Wolters EC, van Wijngaarden GK, Stam FC, et al. Leucoencephalopathy after inhaling "heroin" pyrolysate. Lancet 1982;2:1233-37 Medline

20. Akiba T, Okeda R, Tajima T. Metabolites of 5-fluorouracil, alphafluoro-beta-alanine and fluoroacetic acid, directly injure myelinated fibers in tissue culture. Acta Neuropathol 1996;92:8-13 CrossRef Medline

21. Shibutani M, Okeda R. Experimental study on subacute neurotoxicity of methotrexate in cats. Acta Neuropathol 1989;78:291-300 CrossRef Medline
22. Okeda R, Shibutani M, Matsuo T, et al. Experimental neurotoxicity of 5-fluorouracil and its derivatives is due to poisoning by the monofluorinated organic metabolites, monofluoroacetic acid and alpha-fluoro-beta-alanine. Acta Neuropathol 1990;81:66-73 CrossRef Medline

23. Aydin K, Donmez F, Tuzun U, et al. Diffusion MR findings in cyclosporin-a induced encephalopathy. Neuroradiology 2004;46: 822-24 CrossRef Medline

24. Wilasrusmee C, Da Silva M, Singh B, et al. Morphological and biochemical effects of immunosuppressive drugs in a capillary tube assay for endothelial dysfunction. Clin Transplant 2003;17 (Suppl 9):6-12 CrossRef Medline

25. Lee MS, McKinney AM, Brace JR, et al. Clinical and imaging features of fludarabine neurotoxicity. J Neuroophthalmol 2010;30:37-41 CrossRef Medline

26. Kaiko RF, Wallenstein SL, Rogers A, et al. Relative analgesic potency of intramuscular heroin and morphine in cancer patients with postoperative pain and chronic pain due to cancer. NIDA Res Monogr 1981;34:213-19 Medline

27. Offiah C, Hall E. Heroin-induced leukoencephalopathy: characterization using MRI, diffusion-weighted imaging, and MR spectroscopy. Clin Radiol 2008;63:146-52 CrossRef Medline

28. Rykken JB, McKinney AM. Posterior reversible encephalopathy syndrome. Semin Ultrasound CT MR 2014;35:118-35 CrossRef Medline

29. Jeon SB, Sohn CH, Seo DW, et al. Acute brain lesions on magnetic resonance imaging and delayed neurological sequelae in carbon monoxide poisoning. JAMA Neurol 2018;75:436-43 CrossRef Medline

30. Benson JC, Payabvash S, Thalken GL, et al. Delineation of microhemorrhage in acute hepatic encephalopathy using susceptibilityweighted imaging. Eur J Radiol 2016;85:629-34 CrossRef Medline

31. Boggs JM. Myelin basic protein: a multifunctional protein. Cell Mol Life Sci 2006;63:1945-61 CrossRef Medline

32. Whitaker JN. Myelin basic protein in cerebrospinal fluid and other body fluids. Mult Scler 1998;4:16-21 Medline

33. Fan R, Schrott LM, Arnold T, et al. Chronic oxycodone induces axonal degeneration in rat brain. BMC Neurosci 2018;19:15 CrossRef Medline

34. Kim DM, Lee IH, Song CJ. Uremic encephalopathy: MR imaging findings and clinical correlation. AJNR Am J Neuroradiol 2016;37: 1604-09 CrossRef Medline

35. Kang E, Jeon SJ, Choi SS. Uremic encephalopathy with atypical magnetic resonance features on diffusion-weighted images. Korean $J$ Radiol 2012;13:808-11 CrossRef Medline

36. Drew DA, Koo BB, Bhadelia $\mathrm{R}$, et al. White matter damage in maintenance hemodialysis patients: a diffusion tensor imaging study. BMC Nephrol 2017;18:213 CrossRef Medline

37. Bugnicourt JM, Godefroy O, Chillon JM, et al. Cognitive disorders and dementia in CKD: the neglected kidney-brain axis. J Am Soc Nephrol 2013;24:353-63 CrossRef Medline 